\title{
Digestion ruminale et absorption intestinale des protéines du lupin extrudé chez la vache laitière
}

\author{
C Benchaar, C Bayourthe, R Moncoulon, M Vernay * \\ ENSAT, laboratoire de zootechnie et des productions animales, 145 av de Muret, \\ 31076 Toulouse Cedex, France
}

(Reçu le 9 avril 1991; accepté le 28 août 1991)

\begin{abstract}
Résumé - Quatre vaches Holstein en lactation munies de canules (rumen, duodénum et iléon) sont utilisées afin d'étudier l'effet de l'extrusion à $195^{\circ} \mathrm{C}$ sur le devenir des matières organique et azotée de la graine entière du lupin (Lupinus albus cv Lublanc). Le Cr-EDTA et $\mathrm{YbCl}_{3}$ sont utilisés comme marqueurs respectifs des phases liquide et solide. Les protéines bactériennes sont estimées par le biais des bases puriques et de ${ }^{15} \mathrm{~N}$. L'incorporation du lupin extrudé dans la ration des animaux ne modifie pas l'orientation des fermentations et la digestion apparente de la matière organique dans le rumen; par contre, elle diminue la dégradation des matières azotées dans la panse et de ce fait augmente d'une part, la quantité de protéines d'origine alimentaire à l'entrée du duodénum et, d'autre part, leur digestibilité dans l'intestin grêle (l'arrivée de l'azote bactérien dans le duodénum n'étant pas modifiée). L'ingestion de lupin extrudé n'affecte pas la digestibilité des matières organique et azotée dans l'ensemble du tube digestif.
\end{abstract}

lupin / extrusion / protéines / rumen / intestin

Summary - Ruminal digestion and intestinal absorption of extruded lupin seeds in lactating cows. Four lactating cows fitted with permanent ruminal, duodenal and ileal cannulae were used to study the effect of extrusion of whole lupin seeds at $195^{\circ} \mathrm{C}$ (Lupinus albus $\mathrm{cv}$ Lublanc) on organic matter (OM) and nitrogen $(N)$ degradation in the rumen and their flow to and absorption from the small intestine. Raw whole lupin seeds (RWLS) and extruded whole lupin seeds (EWLS) were fed in diets containing $15.5 \%$ crude protein and composed of $22.6 \%$ whole lupin seeds, $56.5 \%$ corn silage, $10.2 \%$ corn grain and $10.7 \%$ Italian ray-grass on a DM basis, supplemented with vitamins and minerals. Chromium ethylenediaminotetraacetic (Cr-EDTA) and ytterbium chloride $\left(\mathrm{YbCl}_{3}\right)$ were used as liquid and particulate markers respectively, while purines and ${ }^{15} \mathrm{~N}$ ammonium sulfate were utilized as bacterial markers. Cows fed EWLS had a similar ruminal ammonia $N$ and volatile fatty acid concentrations and efficiency of bacterial protein synthesis compared to those fed the RWLS diet. Total tract $O M$ and N digestion were not affected by inclusion of EWLS instead of RWLS; the corresponding mean values were 70 and $71 \%$. Apparent degradation of $O M$ and $N$ in the rumen were 44 and $64 \%$ for diets containing RWLS, and 40 and $39 \%$ for EWLS diets. Feeding diets including EWLS both increased non ammonia $N$ and dietary $N$ flow to the duodenum compared with diets containing RWLS (472 vs $357 \mathrm{~g} / d$ ) and (263 vs $153 \mathrm{~g} / d$ ) respectively. Absorption from the small intestine ( $\mathrm{g} / \mathrm{d}$ and \% entering) of dietary $N$ was higher for EWLS diets (146 vs $62 \mathrm{~g} / d ; 34 \mathrm{vs} 15 \%$ ). The PDIA, PDIE and PDIN contents ( $\mathrm{g} / \mathrm{kg}$ of $D M$ ) of RWLS were 18, 94 and 245 respectively; the corresponding values after extrusion were 145, 220 and 220 .

\section{lupin seed / extrusion / protein / rumen / intestine}

\footnotetext{
* Correspondance et tirés à part
} 


\section{INTRODUCTION}

Le lupin blanc doux est une légumineuse dont les graines sont bien pourvues en protéines : $36 \%$ de la matière sèche (MS) d'après Guillaume et al (1987). De ce fait, son utilisation dans la ration des ruminants présente un intérêt pour les animaux forts producteurs. Or, des valeurs élevées de dégradabilité ruminale $(80-95 \%)$, dues à une forte solubilité de l'azote $(\mathrm{N})$, ont été signalées pour cette graine (Freer et Dove, 1984; Guillaume et al, 1987; Valentine et Bartsch, 1988). En conséquence, ce protéagineux ne pourrait être substitué aux tourteaux et graines de soja dans l'alimentation des vaches laitières qu'après traitement technologique. Au cours d'un précédent travail, nous avons pu montrer que la cuisson-extrusion à 120, 150 et $195^{\circ} \mathrm{C}$ de la graine broyée de lupin (Lupinus albus $c v$ Lublanc) insolubilise les protéines, diminue leur dégradabilité dans le rumen et augmente leur disponibilité intestinale (Cros et al, 1991a). Ces résultats ayant été obtenus in sacco dans le rumen et en sachets mobiles dans les régions postérieures du tube digestif, nous n'avons pu évaluer les flux aux niveaux duodénal et iléal et donc confirmer l'hypothèse d'un effet protecteur de l'extrusion sur les matières azotées (MẢ) de cette graine.

La présente étude a pour but de déterminer l'incidence de l'extrusion à $195{ }^{\circ} \mathrm{C}$ de la graine de lupin sur le devenir de la matière organique $(\mathrm{MO})$ et des $\mathrm{MA}$ : dégradation ruminale, flux duodénal et iléal et absorption intestinale.

\section{MATÉRIEL ET MÉTHODES}

\begin{abstract}
Animaux
Quatre vaches laitières de race Holstein en lactation $(35 \mathrm{~kg} / \mathrm{j})$ pesant environ $650 \mathrm{~kg}$ et munies de canules (rumen, duodénum proximal et iléon terminal) ont été utilisées pour cette expérimentation.
\end{abstract}

\section{Régimes alimentaires}

Au cours de 2 expérimentations (espacées de 3 semaines), les animaux ont reçu quotidiennement en 4 repas égaux $(6,12,18$ et $24 \mathrm{~h})$ une ration composée, en $\mathrm{kg}$ de MS, d'ensilage de maïs plante entière (10), de foin de ray-grass d'Italie $(1,9)$ et de maïs grain broyé $(1,8)$. En outre, toutes les vaches ont reçu $4 \mathrm{~kg}$ de graines de lupin broyées : crues (LC) ou extrudées $^{*}$ (LE) à $195^{\circ} \mathrm{C}$, et $50 \mathrm{~g}$ d'un complexe ${ }^{* *}$ de minéraux et de vitamines. Les rations ont été formulées de telle sorte que $50 \%$ des MA soient apportées par LC ou LE. La composition des différents aliments utilisés est consignée dans le tableau $\mathrm{I}$.

\section{Protocole expérimental}

Le chrome éthylènediaminotétraacétique : $\mathrm{Cr}$ EDTA (Binnerts et al, 1968) et le chlorure d'ytterbium : $\mathrm{YbCl}_{3}$ (Ellis et al, 1982) ont été utilisés respectivement comme marqueurs des phases liquide et solide. Les protéines bactériennes ont été identifiées par le biais des bases puriques et du sulfate d'ammonium ( $\left.{ }^{(5} \mathrm{NH}_{4}\right)_{2} \mathrm{SO}_{4}$.

Après 15 j $\left(J_{1}-J_{15}\right)$ d'adaptation à la ration (LC ou LE), l'expérimentation a débuté, pour

- Extrudeur bivis Werner et Pfleiderer (Continua 37)

** Minéraux (\%): $\mathrm{P}(5), \mathrm{Ca}(14), \mathrm{Na}(6), \mathrm{Mg}(6), \mathrm{Zn}(0,4), \mathrm{Mn}(0,32), \mathrm{Fe}(0,3), \mathrm{Cu}(0,08)$; vitamines (Ul/ $\mathrm{kg})$ : A (250 800), D3 (62 700), E (112) 
Tableau I. Matière sèche (g/kg PF), composition ( $g / \mathrm{kg} \mathrm{MS)} \mathrm{et} \mathrm{valeur} \mathrm{énergétique} \mathrm{(Mcal} / \mathrm{kg} \mathrm{MS}$ ) des differents aliments.

\begin{tabular}{lrrrrrrr}
\hline Aliments & MS & MO & MA & NDF & \multicolumn{1}{c}{ ADF } & \multicolumn{1}{l}{$L$} & EB \\
\hline Ensilage de maïs & 334,0 & 949,0 & 103,0 & 470,0 & 212,0 & 38,0 & 4,4 \\
Foin de ray-grass & 922,2 & 926,0 & 58,7 & 688,0 & 393,0 & 92,0 & 4,3 \\
Maïs & 883,8 & 986,1 & 84,9 & 129,0 & 25,7 & 6,8 & 4,5 \\
Lupin : cru & 899,0 & 961,7 & 363,8 & 176,2 & 154,0 & 53,0 & 5,0 \\
$\quad$ extrudé & 923,7 & 958,0 & 366,8 & 195,1 & 145,6 & 34,0 & $\mathbf{5 , 0}$ \\
\hline
\end{tabular}

Matières : sèche (MS), organique (MO) et azotée (MA) ; neutral (NDF) et acid (ADF) detergent fiber. Lignine (LI) et énergie brute (EB).

chaque animal, par l'administration, via la canule ruminale, d'une dose unique de Cr-EDTA $(1,4 \mathrm{~g} \mathrm{Cr})$ et de $\mathrm{YbCl}_{3}(2 \mathrm{~g} \mathrm{Yb})$. Au cours des $7 \mathrm{j}$ suivants $\left(J_{15}-J_{22}\right)$ les solutions de marqueurs ont été infusées en continu* dans la panse soit, $(\mathrm{g} / \mathrm{j}): 2,77$ de $\mathrm{Cr}, 2$ de $\mathrm{Yb}$ et 0,55 de ${ }^{15} \mathrm{~N}$. Entre $J_{19}$ et $J_{24}$ les prélèvements de digesta : ruminal, duodénal et iléal, les mesures des quantités de MS volontairement ingérées, de même que la récolte totale des fèces ont été effectués pour chacune des 2 périodes expérimentales.

\section{Prélèvements et traitement des digesta}

Dans un premier temps et afin d'évaluer les flux digestifs, des prélèvements de matériel duodénal $(250 \mathrm{ml})$ et iléal $(100 \mathrm{ml})$ ont été effectués pendant 3 jours successifs $\left(J_{19}-J_{22}\right)$, à raison de 4 prélèvements par jour. Après homogénéisation, les digesta duodénaux ont été fractionnés selon la méthode de double marquage préconisée par Faichney (1980). Dans un deuxième temps $\left(J_{22}-J_{24}\right)$ du matériel ruminal a été prélevé (2 $L ; 15$ min avant, 1,2 et $4 \mathrm{~h}$ après les repas de 6 et $18 \mathrm{~h}$ ) afin de déterminer les paramètres fermentaires. Après mesure du $\mathrm{pH}^{* *}$, le fluide ruminal a été filtré à travers un tamis $(1 \mathrm{~mm}$ de maille). Le filtrat obtenu a été fractionné en vue

\footnotetext{
* Pompe péristaltique, 4 canaux (Gilson, France)

** pH-mètre Tacussel, Minisil 6.000
}

des dosages d'ammoniac $\left(\mathrm{N}-\mathrm{NH}_{3}\right)$ et d'acides gras volatils (AGV), et de l'isolement du culot bactérien (Jouany et Thivend, 1972). Au fur et à mesure de leur obtention, les filtrats ruminaux, les fractions duodénales et le matériel iléal ont été regroupés par catégorie et congelés à $-20^{\circ} \mathrm{C}$ jusqu'au moment des analyses. Enfin, la totalité des fèces a été récoltée $\left(J_{19}-J_{24}\right)$ en vue de l'évaluation de la digestibilité des principaux constituants de la ration d'une part et du taux de récupération des marqueurs infusés d'autre part.

\section{Analyses}

Les teneurs en MS ont été déterminées par séchage à l'étuve $\left(105^{\circ} \mathrm{C} ; 24 \mathrm{~h}\right)$, les cendres ont été obtenues par incinération des échantillons secs au four à moufle $\left(550^{\circ} \mathrm{C} ; 12 \mathrm{~h}\right)$; la perte de poids observée représente la MO. Sur les MS ingérées et excrétées, l'énergie brute a été estimée à la bombe calorique adiabatique (Gallenkamp) et les glucides membranaires ont été dosés selon la méthode préconisée par Van Soest (1963) et Van Soest et Wine (1967; 1968). L'azote total (Nt) des aliments, des refus, des fèces, des fluides digestifs et des bactéries a été évalué selon une technique dérivant de 
celle de Kjeldahl. La solubilité de $\mathrm{N}$ des protéines du lupin (LC et LE) a été déterminée in vitro (Vérité et Demarquilly, 1978). La concentration en $\mathrm{N}-\mathrm{NH}_{3}$ des milieux digestifs a été mesurée selon la procédure de Verdouw et al (1977); l'analyse des AGV a été réalisée par chromatographie en phase gazeuse* d'après la technique décrite par Jouany (1982). Les teneurs en bases puriques et en ${ }^{15} \mathrm{~N}$ des bactéries et des digesta intestinaux lyophilisés ont été respectivement déterminées selon les procédés de Ushida et al (1985) et de Al Rabbat et al (1971); ${ }^{15} \mathrm{~N}$ a été dosé par spectrophotométrie de masse*. A partir des échantillons digestifs secs, $\mathrm{Cr}$ a été extrait selon la technique de Siddons et al (1985). L'extraction de Yb a été réalisée d'après la méthode d'Ellis et al (1982). Ces 2 éléments ont été dosés par spectrophotométrie d'absorption atomique ${ }^{* * *}$ à l'aide d'une flamme air-acétylène pour le premier et d'un mélange protoxyde d'azote-acétylène pour le second.

\section{Calculs}

Les flux intestinaux des différents constituants, corrigés par les taux de récupération des marqueurs dans les fèces ont été calculés par double marquage (Faichney, 1980) pour le duodénum (matériel hétérogène) et par simple marquage pour l'iléon. Les taux de récupération (\%) du $\mathrm{Cr}$ et de $\mathrm{Yb}$ ont été de 94,3 $\pm 1,9$ et de 87,1 $\pm 2,2$ respectivement avec la ration supplémentée en LC; les valeurs correspondantes pour le régime LE ont été : $87,5 \pm 2,4$ et $95,9 \pm 6,1$. Les proportions de $\mathrm{N}$ bactérien dans $\mathrm{Nt}$ intestinal ont été calculées en établissant le rapport suivant : (marqueur bactérien/Nt intestinal) / (marqueur bactérien/Nt bactérien). Les quantités de MO réellement dégradées dans le rumen (MORDR) ont été évaluées en déduisant la MO d'origine bactérienne du flux duodénal.

Chaque donnée analytique représente la moyenne arithmétique des échantillons affectée de l'erreur standard $(m \pm S E$ ). L'exploitation statistique des résultats a été effectuée à l'aide du test $t$ de Student au seuil de $P<0,05$.

\section{RÉSULTATS}

\section{Régimes}

Les rations proposées aux vaches en lactation étaient isoazotées $(15,5 \% \mathrm{MA})$ et isoénergétiques (4,5 Mcal $/ \mathrm{kg} \mathrm{MS})$. L'incorporation de LC ou de LE au régime n'a pas significativement affecté l'ingestion volontaire des animaux expérimentaux et la digestibilité des principaux constituants de la ration (tableau II).

\section{Solubilité de l'azote in vitro}

Si la teneur en $\mathrm{N}$ des graines de lupin n'est pas significativement influencée par l'extrusion (tableau I), il n'en est pas de même pour sa solubilité. Après traitement la frac-

Tableau II. Influence de la nature des graines de lupin (crues ou extrudées à $195^{\circ} \mathrm{C}$ ) sur la digestibilité des principaux constituants de la ration.

\begin{tabular}{lcc}
\hline & $L C$ & $L E$ \\
\hline $\begin{array}{l}\text { Quantités ingérées } \\
\text { (kg MS/) }\end{array}$ & $17,5^{\mathrm{a}} \pm 0,4$ & $17,2^{\mathrm{a}} \pm 0,6$ \\
$\begin{array}{l}\text { Digestibilités (\%) } \\
\text { MO }\end{array}$ & & \\
MA & $70,3^{\mathrm{a}} \pm 0,7$ & $68,7^{\mathrm{a}} \pm 0,4$ \\
NDF & $71,9^{\mathrm{a}} \pm 1,2$ & $70,9^{\mathrm{a}} \pm 1,1$ \\
ADF & $51,3^{\mathrm{a}} \pm 0,9$ & $51,3^{\mathrm{a}} \pm 2,2$ \\
EB & $50,5^{\mathrm{a}} \pm 0,2$ & $50,9^{\mathrm{a}} \pm 0,9$ \\
& $68,9^{\mathrm{a}} \pm 1,0$ & $67,2^{\mathrm{a}} \pm 0,7$ \\
\hline
\end{tabular}

Moyenne \pm SE; graines de lupin : crues (LC) ou extrudées (LE). Matières : sèche (MS), organique (MO) et azotée (MA) ; neutral (NDF) et acid (ADF) detergent fiber. Énergie brute (EB). Les valeurs moyennes sur une méme ligne affectées d'un mème exposant ne sont pas statistiquement différentes $(P<0,05)$.

\footnotetext{
* Intersmat; IGC $120 \mathrm{FB}$

"* VG instrument $903 \mathrm{EF}$

*** Perkin Elmer 3086
} 
tion $\mathrm{N}$ soluble est considérablement diminuée (22 vs $77 \% \mathrm{Nt}$ ).

\section{Paramètres fermentaires}

L'ingestion des rations LC et LE n'a pas significativement modifié les paramètres fermentaires des animaux expérimentaux (tableau III). En effet, quel que soit le régime ingéré, le $\mathrm{pH}$ ruminal est demeuré relativement constant $(6,3)$ de même que les concentrations en $\mathrm{N}-\mathrm{NH}_{3}(154 \mathrm{mg} / \mathrm{l})$ et en AGV (108 mmol/l). Par ailleurs, la répartition qualitative de ces AGV n'a pas été influencée.

Tableau III. Influence de la nature des graines de lupin (crues ou extrudées à $195^{\circ} \mathrm{C}$ ) sur les paramètres fermentaires ruminaux.

\begin{tabular}{lcc}
\hline $\begin{array}{l}\text { Paramètres } \\
\text { fermentaires }\end{array}$ & $L C(64)$ & $L E(64)$ \\
\hline $\mathrm{pH}$ & $6,2^{\mathrm{a}} \pm 0,1$ & $6,4^{\mathrm{a}} \pm 0,1$ \\
$\mathrm{~N}-\mathrm{NH}_{3}(\mathrm{mg} / \mathrm{l})$ & $159,0^{\mathrm{a}} \pm 10,1$ & $148,0^{\mathrm{a}} \pm 8,2$ \\
$\mathrm{AGVt}(\mathrm{mmol} / \mathrm{l})$ & $106,5^{\mathrm{a}} \pm 2,9$ & $109,8^{\mathrm{a}} \pm 5,8$ \\
$\mathrm{AGV}(\% \mathrm{AGV})$ & & \\
& & \\
Acétate & $59,8^{\mathrm{a}} \pm 0,5$ & $60,9^{\mathrm{a}} \pm 0,8$ \\
Proprionate & $22,5^{\mathrm{a}} \pm 0,8$ & $21,1^{\mathrm{a}} \pm 1,0$ \\
Isobutyrate & $0,9^{\mathrm{a}} \pm 0,04$ & $0,7^{\mathrm{a}} \pm 0,04$ \\
Butyrate & $12,2^{\mathrm{a}} \pm 0,3$ & $13,4^{\mathrm{a}} \pm 0,2$ \\
lsovalérate & $2,4^{\mathrm{a}} \pm 0,3$ & $2,0^{\mathrm{a}} \pm 0,1$ \\
Valérate & $1,6^{\mathrm{a}} \pm 0,3$ & $1,8^{\mathrm{a}} \pm 0,1$ \\
& & \\
\hline
\end{tabular}

Moyenne $\pm S E$; nombre d'échantillons entre parentheses; graines de lupin : crues (LC) ou extrudées (LE). Azote ammoniacal $\left(\mathrm{N}-\mathrm{NH}_{3}\right)$ et acides gras volatils totaux (AGVt). Les valeurs moyennes sur une même ligne affectées d'un méme exposant ne sont pas statistiquement différentes $(P<0,05)$.

\section{Flux et digestion de la matière organique}

De l'examen du tableau IV, il ressort que les flux de MO, au niveau des différents segments du tube digestif n'ont pas été si-
Tableau IV. Ingestion, flux et digestion de la matière organique dans le tube digestif de vaches recevant une ration complémentée en graines de lupin (crues ou extrudées à $195^{\circ} \mathrm{C}$ ).

\begin{tabular}{lrr} 
Matière organique & \multicolumn{1}{c}{$L C$} & \multicolumn{1}{c}{$L E$} \\
\hline Ingestion (kg/) & $16,5^{\mathrm{a}} \pm 0,7$ & $16,3^{\mathrm{a}} \pm 0,8$ \\
& & \\
Flux (kg/j) & & \\
Duodénal & $9,3^{\mathrm{a}} \pm 0,4$ & $9,9^{\mathrm{a}} \pm 0,4$ \\
lléal & $6,0^{\mathrm{a}} \pm 0,5$ & $5,6^{\mathrm{a}} \pm 0,2$ \\
Fécal & $4,9^{\mathrm{a}} \pm 0,1$ & $5,1^{\mathrm{a}} \pm 0,2$ \\
& & \\
Digestion ruminale & & \\
Apparente (\% ingéré) & $43,6^{\mathrm{a}} \pm 0,4$ & $39,9^{\mathrm{a}} \pm 3,7$ \\
Réelle (kg/j) & $9,6^{\mathrm{a}} \pm 0,6$ & $9,3^{\mathrm{a}} \pm 1,0$ \\
Réelle (\% ingéré) & $58,2^{\mathrm{a}} \pm 1,2$ & $57,1^{\mathrm{a}} \pm 3,6$ \\
& & \\
Absorption intestinale & $19,5^{\mathrm{a}} \pm 3,1$ & $26,5^{\mathrm{a}} \pm 3,2$ \\
(\% ingéré) & &
\end{tabular}

Moyenne $\pm S E$; graines de lupin : crues $(L C)$ ou extrudées (LE). Les valeurs moyennes sur une même ligne affectées d'un même exposant ne sont pas statistiquement différentes $(P<0,05)$.

gnificativement modifiés par la substitution de LC par LE dans la ration. Cependant la digestion ruminale apparente de la $\mathrm{MO}$ était légèrement plus faible $(-8 \%)$ et l'absorption intestinale plus élevée (+ $36 \%)$, mais ces variations n'étaient pas significatives. De même, lorsque la participation des micro-organismes ruminaux est prise en considération, il apparaît que la digestion réelle de la MO au niveau des réservoirs de fermentation n'a pas été significativement affectée par l'ingestion de la ration $\mathrm{LE}$, soit une moyenne de $9,5 \mathrm{~kg} / \mathrm{j}$.

\section{Flux et digestion de la matière azotée}

Pour les différentes formes azotées, les flux intestinaux sont consignés dans le tableau V. Au niveau duodénal, les flux ( $g / j)$ 
de Nt et de $\mathrm{N}$ non ammoniacal étaient significativement plus élevés lorsque les vaches ingéraient la ration supplémentée en LE, soit une hausse de $32 \%$ environ pour chacun des flux. Notons qu'à ce niveau $\mathrm{Nt}$ représente $113 \%$ de $\mathrm{N}$ ingéré pour LE et $86 \%$ pour le régime LC. Par contre, le flux de $\mathrm{N}$ bactérien est demeuré inchangé, (206 g/j); en conséquence, la quantité de $\mathrm{N}$ alimentaire parvenant au duodénum a significativement augmenté $(+72 \%)$. Le pourcentage de $\mathrm{N}$ alimentaire apparemment dégradé dans le réticulorumen est alors passé de $64 \%$ de $N$ ingéré

Tableau V. Ingestion, flux et digestion des matières azotées dans le tube digestif de vaches recevant une ration complémentée en graines de lupin (crues ou extrudées à $195^{\circ} \mathrm{C}$ ).

\begin{tabular}{|c|c|c|}
\hline Matières azotées & $L C$ & $L E$ \\
\hline Ingestion $(g / j)$ & $427,3^{a} \pm 10,9$ & $430,0^{a} \pm 14,1$ \\
\hline \multicolumn{3}{|l|}{ Flux $(g / j)$} \\
\hline $\begin{array}{l}\text { Nt duodénal } \\
\mathrm{N} \text { non ammoniacal } \\
\mathrm{N} \text { bactérien* } \\
\mathrm{N} \text { alimentaire }\end{array}$ & $\begin{array}{l}369,1^{a} \pm 19,7 \\
357,1^{a} \pm 18,2 \\
203,9^{a} \pm 7,5 \\
153,1^{a} \pm 10,7\end{array}$ & $\begin{array}{l}485,8^{b} \pm 8,4 \\
471,7^{b} \pm 6,2 \\
208,3^{a} \pm 8,8 \\
263,3^{b} \pm 2,7\end{array}$ \\
\hline $\begin{array}{l}\text { Nt iléal } \\
\mathrm{N} \text { non ammoniacal } \\
\mathrm{N} \text { bactérien } \\
\mathrm{N} \text { alimentaire }\end{array}$ & $\begin{array}{r}144,7^{\mathrm{a}} \pm 4,0 \\
140,5^{\mathrm{a}} \pm 3,6 \\
48,9^{\mathrm{a}} \pm 7,4 \\
91,4^{\mathrm{a}} \pm 3,7\end{array}$ & $\begin{array}{r}164,7^{\mathrm{a}} \pm 6,5 \\
160,5^{\mathrm{a}} \pm 6,0 \\
43,4^{\mathrm{a}} \pm 4,5 \\
117,1^{\mathrm{b}} \pm 1,5\end{array}$ \\
\hline Nt fécal & $120,3^{a} \pm 2,0$ & $125,0^{b} \pm 0,6$ \\
\hline $\begin{array}{l}\text { Digestion ruminale apparente } \\
\text { de } N \text { alimentaire (\% ingéré) }\end{array}$ & $64,1^{a} \pm 3,4$ & $38,7^{b} \pm 2,6$ \\
\hline $\begin{array}{l}\text { Synthèse bactérienne }(g \mathrm{~N} / \mathrm{kg}) \\
\text { MOADR } \\
\text { MORDR }\end{array}$ & $\begin{array}{l}28,3^{a} \pm 1,8 \\
20,7^{a} \pm 1,5\end{array}$ & $\begin{array}{l}33,1^{a} \pm 3,4 \\
22,6^{a} \pm 1,4\end{array}$ \\
\hline \multicolumn{3}{|l|}{ Absorption intestinale } \\
\hline $\begin{array}{l}\text { N non ammoniacal }(g / j) \\
\text { (\% ingéré) } \\
(\% \text { entrée) }\end{array}$ & $\begin{array}{r}216,6^{a} \pm 7,4 \\
50,9^{a} \pm 3,2 \\
60,6^{a} \pm 1,0\end{array}$ & $\begin{array}{r}311,2^{b} \pm 0,2 \\
72,9^{b} \pm 0,6 \\
65,9^{b} \pm 0,8\end{array}$ \\
\hline $\begin{array}{l}N \text { alimentaire }(g / j) \\
(\% \text { ingéré) } \\
(\% \text { entrée) }\end{array}$ & $\begin{array}{l}61,7^{a} \pm 7,1 \\
14,5^{a} \pm 2,6 \\
39,9^{a} \pm 4,7\end{array}$ & $\begin{array}{r}146,2^{\mathrm{b}} \pm 4,2 \\
34,0^{\mathrm{b}} \pm 1,5 \\
53,9^{\mathrm{b}} \pm 1,0\end{array}$ \\
\hline
\end{tabular}

Moyenne $\pm S E$; graines de lupin : crues (LC) ou extrudées (LE); azote total (Nt). * moyenne des valeurs ARN et ${ }^{15} \mathrm{~N}_{;}{ }^{* *}=\mathrm{N}$ alimentaire + endogène. Matière organique apparemment (MOADR) ou réellement (MORDR) dégradée dans le rumen. Les valeurs moyennes sur une même ligne affectées d'un méme exposant ne sont pas statistiquement differentes $(P<0,05)$. 
(pour la ration complémentée en LC) à $39 \%$ (pour la ration contenant LE) soit une diminution de $40 \%$ environ. Au cours du transit intestinal, l'absorption journalière de $\mathbf{N}$ d'origine alimentaire a été significativement plus importante pour les vaches ayant accès au régime LE; l'augmentation enregistrée est en moyenne de $137 \%$. Par ailleurs, l'extrusion du lupin a significativement amélioré les digestibilités intestinales de $\mathrm{N}$ alimentaire $(+35 \%)$ et de $\mathrm{N}$ non ammoniacal $(+9 \%)$. L'absorption intestinale de $\mathrm{N}$ bactérien, de même que l'efficacité de la synthèse bactérienne, n'ont pas été significativement modifiées par le changement de ration (LC ou LE), soit environ $78 \%$ et $22 \mathrm{~g} / \mathrm{kg}$ MORDR respectivement. Le remplacement de $L C$ dans la ration par LE a également entraîné une hausse du flux iléal de $\mathrm{Nt}(+14 \%)$ et de $\mathrm{N}$ alimentaire $(+28 \%)$, le flux fécal n'étant pas modifié; la digestion caecocolique des MA a donc été accrue (9 vs $6 \%$ ingéré).

\section{DISCUSSION}

L'extrusion à haute température $\left(195^{\circ} \mathrm{C}\right)$ de la graine entière du lupin a provoqué une forte diminution de la solubilité de l'azote en salive artificielle, résultat qui est en accord avec ceux obtenus par de nombreux chercheurs sur d'autres graines traitées thermiquement, ie soja (Stern et al, 1985; Michalet-Doreau et al, 1985), coton (Arieli et al, 1989) ou féverole (Cros et al, 1991b).

Au niveau de la digestion ruminale, la substitution de LC par LE dans la ration n'a pas modifié l'orientation des fermentations. Paradoxalement, bien que $\mathrm{N}$ de $\mathrm{LC}$ soit plus soluble et donc plus dégradable que $\mathrm{N}$ de LE, la production de $\mathrm{N}-\mathrm{NH}_{3}$ n'a pas été réduite comme on pouvait s'y attendre d'après les résultats des expérimentations de Block et al (1981), de Pena et al (1986) et de Focant et al (1990). Dans nos condi- tions expérimentales, l'extrusion ne semble donc pas avoir d'incidence sur ce paramètre, des faits similaires ont été obtenus par d'autres équipes (McMeniman et Armstrong, 1979; Michalet-Doreau et al, 1985; Stern et al, 1985) avec les tourteaux et graines (soja, féverole) traités thermiquement. Par ailleurs, l'efficacité de la synthèse bactérienne, lorsqu'elle est exprimée en g de N/kg MORDR, n'a pas été affectée par l'extrusion de la graine de lupin. La valeur que nous obtenons pour cette expérimentation (22) est proche de celles mentionnées par Zerbini et al (1988) chez des vaches en lactation ingérant des rations à base d'ensilage de maïs (25), et par McMeniman et Armstrong (1979) pour des vaches recevant un régime supplémenté en féverole traitée à la chaleur (21). Toutefois, une donnée nettement supérieure (42) est signalée par Santos et al (1984) lorsque les animaux sont alimentés avec des rations à base d'ensilage de maïs.

L'extrusion n'a pas modifié la digestibilité totale des différents constituants de la ration. Toutefois, il convient de noter que la valeur de digestibilité de la fraction pariétale que nous obtenons (51\%) est plus faible que celles relevées dans la littérature $(60 \%)$ pour des régimes comparables. La richesse en MG de la ration $(7 \%)$ pourrait expliquer cette faible valeur (Doreau et al, 1991). La dégradation apparente de la MO dans le réticulo-rumen n'a pas été affectée par le traitement thermique $(42 \%$ de l'ingéré), résultat qui va dans le sens des données bibliographiques. C'est ainsi que, pour des vaches laitières recevant en complément azoté du tourteau ou de la graine de soja : crue ou extrudée (Santos et al, 1984; Stern et al, 1985), la digestion ruminale apparente de la MO est de l'ordre de $31 \%$, alors que pour une ration supplémentée en pois, Focant et al (1990) obtiennent des valeurs plus fortes $(51,6$ à $45,4 \%)$ selon que la graine est respectivement crue ou extrudée. Par ailleurs, une valeur 
du même ordre de grandeur que la nôtre (42\%) est donnée par Pena et al (1986) pour un régime renfermant de la graine de coton (crue ou extrudée). Ces fluctuations pourraient être liées aux conditions expérimentales : niveau d'ingestion, nature et proportions des constituants de la ration et au type de marqueur utilisé pour l'évaluation des flux digestifs (Tamminga et al, 1979; McAllan et Smith, 1983; Kung et al, 1983). Au cours du transit dans l'intestin grêle, la digestion apparente de la MO a été discrètement améliorée lorsque les vaches avaient accès à la ration supplémentée en LE. Une hausse de la disparition intestinale de la MO a également été signalée par Pena et al (1986) après addition de graines de coton extrudées à la ration.

Le flux de MA qui parvient au duodénum est la résultante de plusieurs flux : bactérien, ammoniacal, alimentaire et endogène, sur lesquels le traitement thermique peut avoir une incidence. Dans nos conditions expérimentales, la quantité de $\mathrm{N}$ bactérien qui pénètre dans l'intestin grêle n'a pas été modifiée lors de l'ingestion de la ration LE. Des observations comparables sont faites par Stern et al (1985) avec des graines de soja (crues ou extrudées), tandis que d'autres équipes (Pena et al, 1986; Focant et al, 1990) signalent, après extrusion des graines entières de coton et de pois, un accroissement du flux de $\mathrm{N}$ bactérien, et que Rooke et al (1982) enregistrent une réduction de ce flux après traitement du soja par le formaldéhyde. De plus, lorsque les animaux ont eu à leur disposition la ration supplémentée en LE, le flux duodénal de Nt a été d'une part, plus important que chez des vaches consommant la ration $\mathrm{LC}$ et, d'autre part supérieur à $\mathbf{N}$ ingéré. Ce gain azoté, dû au recyclage de l'urée, est très souvent observé pour des régimes apportant moins de $17 \%$ de MA etou à faible teneur en $\mathrm{N}$ fermentescible (Netemeyer et al, 1980; Satter, 1982; Loerch et al, 1983; Santos et al, 1984). C'est ainsi que, pour des régimes comportant des sous-produits industriels (farines de viande et de poisson, tourteau de soja) ou des graines entières de soja extrudées, Loerch et al (1983), Stern et al (1985) et Garrett et al (1987) enregistrent, à la sortie des réservoirs de fermentation, des flux Nt représentant 115 à $132 \%$ de $N$ ingéré. Le recyclage et la diminution de la dégradation des MA dans le rumen ont donc pour conséquence d'augmenter de $72 \%$ la quantité de $\mathrm{N}$ d'origine alimentaire qui parvient à l'intestin grêle. Ceci confirme l'effet protecteur du traitement thermique sur les MA du lupin que nous avions mis en évidence in sacco (Cros et al, 1991a). De même, Stern et al (1985) mentionnent que l'extrusion de la graine de soja à 132 et $149^{\circ} \mathrm{C}$, élève le flux de $\mathrm{N}$ alimentaire de 69 et $100 \%$ respectivement; Pena et al (1986) avec la graine de coton extrudée à $150^{\circ} \mathrm{C}$, obtiennent une augmentation de ce flux de $29 \%$. Au niveau intestinal, le traitement par cuisson-extrusion de la graine entière du lupin a augmenté l'absorption de $\mathrm{N}$ non ammoniacal $(44 \%)$ et plus particulièrement celle de $\mathrm{N}$ d'origine alimentaire (137\%). Par ailleurs, les valeurs que nous enregistrons pour l'absorption de $\mathrm{N}$ non ammoniacal (63\% de l'ingéré) sont proches de celles mentionnées par Van't Klooster et Boekholt (1972), Tamminga (1975) et Zinn et Owens (1983). Dans nos conditions expérimentales il apparaît cependant que le traitement thermique accroît légèrement le flux iléal de $\mathrm{N}$ alimentaire. Au cours du transit dans les régions postérieures, cette fraction supplémentaire est entièrement digérée par la microflore caecale puisque le flux fécal n'est pas modifié.

À partir des résultats obtenus, nous avons évalué les valeurs PDI pour LC et $L E$, en formulant l'hypothèse d'un déficit en énergie avec la ration complémentée en $\mathrm{LC}$ (flux Nt duodénal < à Nt ingéré) et d'un 
déficit en $\mathrm{N}$ soluble lorsque LC est remplacé par LE (flux Nt duodénal > à Nt ingéré). Les valeurs PDI pour l'ensilage de maïs, le foin de ray-grass et le maïs grain ont été prises dans les tables de l'Alimentation de I'INRA (1988). Dans ces conditions, pour la graine crue, les valeurs PDIA, PDIE et PDIN (g/kg MS) sont : 18, 94 et 245 respectivement, les valeurs correspondantes pour LE sont : 145,220 et 220 . II convient de signaler que des données comparables ont été obtenues in situ par Cros et al (1991a) soit : 13, 84 et 224 pour LC et 190,247 et 295 pour LE et par Andrieu et al (1989) pour LC $(13,82,220)$.

II ressort de cette étude que l'incorporation de la graine entière de lupin extrudée à haute température $\left(195^{\circ} \mathrm{C}\right)$ dans les rations de vaches laitieres en lactation ne modifie pas l'orientation des fermentations ruminales. La diminution de la dégradation des MA dans la panse permet d'augmenter la quantité de MA d'origine alimentaire à l'entrée de l'intestin grêle, sans pour autant affecter la part des protéines d'origine bactérienne dans le flux azoté intestinal. De plus, elle améliore la digestibilité intestinale de l'azote alimentaire. Toutefois, pour une meilleure appréciation de l'efficacité du traitement thermique par cuissonextrusion, il conviendrait d'évaluer, aussi, la digestibilité intestinale en termes d'acides aminés réellement digérés et absorbés dans l'intestin grêle.

\section{RÉFÉRENCES}

Al Rabbat MF, Baldwin RL, Weir WC (1971) in vitro ${ }^{15}$ nitrogen tracer technique for some kinetic measures of ruminal ammonia. $J$ Dairy Sci 54, 1150-1161

Andrieu J, Demarquilly C, Sauvant D (1989) Tables of feed used in France. In : Ruminant Nutrition (Jarrige R, ed), INRA, Libbey J, Eurotext, Paris-Londres, 213-294

Arieli A, Ben-Moshe A, Zamwel S, Tagari H (1989) in situ evaluation of the ruminal and intestinal digestibility of heat-treated whole cottonseeds. J Dairy Sci 72, 1228-1233

Binnerts WT, Van't Klooster A Th, Frens AM (1968) Soluble chromium indicator measured by atomic absorption in digestion experiments. Vet Rec 82, 470

Block E, Muller LD, Griel LC Jr, Garwood DL (1981) Brown midrib -3 corn silage and heat extruded soybeans for early lactating dairy cows. J Dairy Sci 64, 1813-1825

Cros $P$, Benchaar $C$, Bayourthe $C$, Vernay $M$, Moncoulon $R$ (1991a) In situ evaluation of the ruminal and intestinal degradability of extruded whole lupin seed nitrogen. Reprod Nutr Dev 31, 575-583

Cros P, Vernay M, Moncoulon R (1991b) In situ evaluation of the ruminal and intestinal degradability of extruded whole horsebeans. Reprod Nutr Dev 31, 249-255

Doreau M, Chilliard Y, Bauchart D, MichaletDoreau B (1991) Influence of different fat supplements on digestibility and ruminal digestion in cows. Ann Zootech 40, 19-30

Ellis WC, Lascano C, Teeter T, Owens FN (1982) Solute and particulate flow markers. In : Protein requirements for cattle. Symposium (FN Owens, ed), Oklahoma State, Univ Stillwater, 37-56

Faichney GJ (1980) Measurement in the sheep of the quantity and composition of rumen digesta and the fractional outflow of digesta constituents. Aust J Agric Res 31, 1129-1137

Focant M, Van Hoecke A, Vanbelle M (1990) The effect of two heat treatments (steam flaking and extrusion) on the digestion of Pisum sativum in the stomachs of heifers. Anim Feod Sci Technol 28, 303-313

Freer $M$, Dove H (1984) Rumen degradation of protein in sunflower meal, rapeseed meal and lupin seed placed in nylon bags. Anim Feed Sci Technol 11, 87-101

Garrett JE, Goodrich RD, Meiske JC, Stern MD (1987) Influence of supplemental nitrogen source on digestion of nitrogen, dry matter and organic matter and on in vivo rate of ruminal protein degradation. J Anim Sci 64, 1801-1812

Guillaume B, Otterby DE, Linn JG, Stern MD, Johnson DG (1987) Comparison of sweet white lupin seeds with soybean meal as a protein supplement for lactating dairy cows. J Dairy Sci 70, 2339-2348

INRA (1988) Tables de l'alimentation des bovins, ovins et caprins, INRA Publ Versailles 
Jouany JP (1982) Volatile fatty acid and alcohol determination, in digestive contents, silage juices, bacterial cultures and anaerobic fermentor contents. Sci Alim 2, 131-144

Jouany JP, Thivend $P$ (1972) Evolution postprandiale de la composition glucidique des corps microbiens du rumen en fonction de la nature des glucides du régime. I. Les protozoaires. Ann Biol Anim Biochim Biophys 12, 673-677

Kung L Jr, Huber JT, Satter LD (1983) Influence of nonprotein nitrogen and protein of low degradability on nitrogen flow and utilization in lactating dairy cows. J Dairy Sci 66, 18631872

Loerch SC, Berger LL, Plegge SD, Fahey GC Jr (1983) Digestibility and rumen escape of soybean meal, blood meal, meat and bone meal and dehydrated alfalia nitrogen. J Anim Sci $57,1037-1047$

McAllan AB, Smith RH (1983) Estimation of flows of organic matter and nitrogen components in post-ruminal digesta and effects of level of dietary intake and physical form of protein supplement on such estimates. $\mathrm{Br} J$ Nutr 49, 119-127

McMeniman NP, Armstrong DG (1979) The flows of amino acids into the small intestine of cattle when fed heated and unheated beans (Vicia faba). J Agric Sci (Camb) 93, 181.188

Michalet-Doreau B, Bogaert C, Beauchard D (1985) Valeur nutritive des graines de soja crues ou extrudées pour les ruminants. Bull Tech CRZV Theix, INRA 59, 29-38

Netemeyer DT, Bush J, Owens FN (1980) Effect of particle size of soybean meal on protein utilization in steers and lactating cows. J Dairy Sci 63, 574-578

Pena F, Tagari H, Satter LD (1986) The effect of heat treatment of whole cottonseed on site and extent of protein digestion in dairy cows. J Anim Sci 62, 1423-1433

Rooke JA, Norton BW, Armstrong DG (1982) The digestion of untreated and formaldehyde treated soybean meals and estimation of their rumen degradabilities by different methods. J Agric Sci (Camb) 99, 441-452

Santos KA, Stern MD, Satter LD (1984) Protein degradation in the rumen and amino acid $a b$ - sorption in the small intestine of lactating dairy cattle fed various protein sources. $J$ Anim Sci 58, 244-255

Satter LD (1982) A metabolisable protein system keyed to ruminal ammonia concentration. The Wisconsin system. In: Protein requirements for cattle symposium (FN Owens, ed) Oklahoma State, Univ Stillwater, 245-264

Siddons RC, Paradine J, Beever DE, Cornell PR (1985) Ytterbium acetate as a particulatephase digesta flow marker. Br J Nutr 54, 509517

Stern MD, Santos KA, Satter LD (1985) Protein degradation in rumen and amino acid absorption in small intestine of lactating dairy cattle fed heat-treated soybeans. J Dairy Sci 68 , 45-56

Tamminga $S$ (1975) The influence of the method of preservation of forages on the digestion in dairy cows. 2. Digestion of organic matter, energy and amino acids in forestomachs and intestines. Neth J Agric Sci 23 , 89-103

Tamminga S, Van Der Koelen CJ, Van Vuuren AM (1979) The effect of the level of feed intake on nitrogen entering the small intestine of dairy cows. Livest Prod Sci 6, 255-261

Ushida K, Lassalas B, Jouany JP (1985) Determination of assay parameters for RNA analysis in bacterial and duodenal samples by spectrophotometry. Influence of sample treatment and preservation. Reprod Nutr Dev 25, 1037-1046

Valentine SC, Bartsch BD (1988) Degradation of dry matter, crude protein, fat, crude fibre and nitrogen-free-extract in milled barley and lupin grains incubated in nylon bags in the rumen of dairy cows. J Agric Sci (Camb) 110, 395-398

Van't Klooster AT, Boekholt HH (1972) Protein digestion in the stomachs and intestines of the cow. Neth J Agric Sci 20, 272-284

Van Soest PJ (1963) Use of detergents in the analysis of fibrous feeds: II. A rapid method for the determination of fiber and lignin. $J$ Assoc Off Agric Chem 46, 829-835

Van Soest PJ, Wine RH (1967) Use of detergents in the analysis of fibrous feeds IV. Determination of plant cell wall constituents. $J$ Assoc Off Agric Chem 50, 50-55 
Van Soest PJ, Wine RH (1968) Determination of lignin and cellulose in acid detergent fiber permanganate. Assoc Off Anal Chem 51, 780-785

Verdouw H, Van Echteld CJA, Dekkers EMJ (1977) Ammonia determination based on indophenol formation within sodium solicylate. Water Res 12, 399-402

Vérité R, Demarquilly C (1978) Qualité des matières azotées des aliments pour ruminants.
In : La vache laitière, INRA, Versailles, 143158

Zerbini E, Polan EC, Herbein JH (1988) Effect of dietary soybean meal and fish meal on protein digesta flow in Holstein cows during early lactation. $J$ Dairy Sci 71, 1248-1258

Zinn RA, Owens FN (1983) Influence of feed intake level on site of digestion in steers fed high concentrate diets. J Anim Sci 56, 471 . 475 